\title{
Adipose Tissue Micrograft in a Scaffold of Plasma-Gel Combined With Platelet-Derived Growth Factors in Dermal Wrinkle Regeneration
}

\author{
Fabiano Svolacchia, ${ }^{1}$ Lorenzo Svolacchia ${ }^{2}$
}

\begin{abstract}
Background: The dermal aging process and the formation of deep wrinkles are a biological involution that also involves the regeneration system of cells immersed in the extracellular matrix and the papillary dermis. The progressive loss of niches of adult stem cells (MSCs) is more evident after the first third of life; it increases the phenotypic expression and the characteristics of the tissue senescence process. The purpose of this study was to clinically demonstrate that in viable micrograft there may be an improvement of deep wrinkles and surrounding tissues.

Methods: This study involved 11 female patients who underwent the correction of deep dermal wrinkles through a suspension containing $0.8 \mathrm{~mL}$ of viable micrografts in a $5 \mathrm{~mL}$ plasma gel scaffold, obtained from the centrifugation of a $20 \mathrm{cc}$ venous sample peripheral blood, gelled by heat in a dry steriliser and the buffy coat coming from the same venous sample, in order verify overtime the improvement of the interested anatomical area. Individual signs of wrinkles and the degree of correction obtained for each treatment and each area were objectively evaluated by using a 10-0 visual analog scale (VAS), Modified Vancouver scale and Berardesca's scale.

Results: With this technique excellent results were obtained. In fact, wrinkles were improved, as well as surrounding tissues, even after 60 days, as shown by the Berardesca's, VAS and Modified Vancouver scales.

Conclusion: This retrospective clinical evaluation allowed us to consider the excellent clinical results obtained with this method for the treatment of deep wrinkles and surrounding tissues, through a suspension of progenitors with MSCs derived from adipose tissue (ADSCa) in a not inflammatory plasma gel scaffold combined with buffy coat.
\end{abstract}

Keywords: Adult Tissue Progenitor; Cluster of Differentiation (CD); Side Population; Buffy coat; PRP, STBA.
(1) Department of Anatomical, Histological, Medical and Legal Sciences and the Locomotor Equipment, Section of Human Anatomy, Laboratory of Experimental Morphology at the University of Rome "La Sapienza", Italy.

(2) Faculty of Medicine and Surgery, University of Rome "La Sapienza", Rome, Italy.

Correspondence:

FABIANO SVOLACCHIA

E: fabiano.svolacchia@gmail.com

\section{ARTICLE INFO}

Received: 14 January 2021 Revision received: 17 February 2021 Accepted: 18 February 2021

\section{Introduction}

Unlike embryonic stem cells (ESCs), adult stem cells (MSCs) have a lower self-maintaining capacity due to lower levels of telomerase activity. ${ }^{1}$ MSCs in adult tissues can be able to avoid the differentiation process which is typical for other cells and to colonise appropriate niches with the function of maintaining their potential in adult life and to limit their differentiation processes. ${ }^{1}$ The loss of dermis tone, with the formation of wrinkles during the aging process, are favored by the worn out of niches of MSCs; ROS and UV of exogenous and endogenous origins are the primary causes of these biological events. ${ }^{2}$ Over time there will be a reduction of natural fibroblasts, with an inver- 
sion of the balance between type III-collagen and type I-collagen, with a decrease in the production of elastin and extracellular matrix (ECM). ${ }^{3}$ Over time, the dermis will be colonised by senescent fibroblasts with poor secretory capacity, which will be directed towards type I or fibrotic collagen. ${ }^{4}$

These pathophysiological events will be implicated in the loss of elasticity of the tissue and in the formation of wrinkles and deep furrows on the face. The positive effects on the use of MSCs in pathologies that involve a dermal regenerative stimulus have already been demonstrated..$^{5}$ It has also been shown that, according to Tonnard 2013, a disaggregated adipose tissue contains viable cells with characteristics of adult stemness; it has been demonstrated that cellular fragments and fibrous shoots contained in a disaggregated adipose according to Tonnard 2013 are potentially inflammatory and that the filtration of disaggregate adipose tissue at 50 microns allows to maintain a vital and numerically high the Side Population (SP). ${ }^{6-8}$ SP in flow cytometry is a sub-population of cells that is distinct from the main population based on the markers employed, cluster of differentiation (CD).

Many recent studies also show that the platelet-rich plasma (PRP) that comes from a centrifuged venous sampling, produces positive effects when it is injected into the dermis, despite the persistence of these effects over time, ${ }^{9}$ in addition, the portion of tissue used for the treatments appears to be in the buffy coat. ${ }^{10}$ Therefore, a portion of autologous plasma for PRP treatments is not used. ${ }^{11}$ This study involved the use of a viable micrograft of adipose tissue containing progenitors in suspension, with markers of adult stem cell on the surface (CD) from $2 \mathrm{~mL}$ of subcutaneous fat, which was taken and disrupted as indicated by Tonnard 2013, then microfiltered through a 50-micron filter combined with a plasma gel scaffold (STBA), suitable for use as an autologous biological tissue support since it is not inflammatory. ${ }^{5-7,13-15}$

It is also envisaged the union of the buffy coat with the suspension obtained immediately before, order allow the physiological activation of progenitors through the surface CDs. ${ }^{7}$ Given the ability of MSCs derived from adipose tissue (ADSCa) to induce physiological neocollagenogenesis, our intention was to clinically verify whether the viable micrograft, mixed with a non-inflammatory
STBA scaffold to which it was added immediately prior to injection into the deep dermis the buffy coat of PRP, could allow a clinical improvement of wrinkles and surrounding tissues, by considering the mechanical protection capacity possessed by a support of autologous gel against the effects of ROS and UV and the properties of the buffy coat. . $^{10,16,17}$

An improvement of the physiological neo-collagenogenesis would be activated through the reaction capacity of the CD 44 present on progenitors, triggered by the PDGF contained in alpha granules of the platelets suspended in the buffy coat, by subsequently improving tissues through the increase of production of elastin, collagen and other components of the extra cytoplasmic matrix. ${ }^{9,10,}$ ${ }^{18,19}$ It is also hypothesized that the expression on the progenitors of other markers such as CD 73, 90 and 105 would affect neo-vasculogenesis and consequently improve the papillary dermis.7, 20 Hypothesis of improving vitality and longer functionality of progenitors, once injected into dermis through this new technique, was linked to the reestablishment of the physiological niches of viable cells, as protected from ROS by an autologous anti-inflammatory biological tissue support (STBA), and physiologically activated by the buffy coat of venous centrifugate. ${ }^{17}$ Therefore, an increase in the number of active and protected progenitors present in dermis would have allowed an increase in biological parameters with a consequent increase in cell turnover with a reduction in deep wrinkles observable in a follow-up.

The study of this method began in 2017 , and it allowed us to clinically evaluate the effects of an increase in the production of elastin, collagen and other components of the extra cytoplasmic matrix, evaluated with the Berardesca's and VAS scales. The Modified Vancouver Scale was also used to evaluate the quality of the tissues adjacent to the imperfections.

\section{Methods}

This study involved 11 female patients who underwent the correction of deep dermal wrinkles through a suspension containing $0.8 \mathrm{~mL}$ of viable micrografts in a $5 \mathrm{~mL}$ plasma gel scaffold, obtained from the centrifugation of a $20 \mathrm{~mL}$ venous sample 
peripheral blood, gelled by heat in a dry steriliser and the buffy coat coming from the same venous sample, in order verify overtime the improvement of the interested anatomical area. All 11 volunteer patients (between 57 and 70 years of age) attended the clinic between 2017 and 2020. To participate in the study, the only obligatory criteria was the presence of deep wrinkles and severe signs of skin aging. The study was performed by following standards of the local ethics committee and in accordance with the Declaration of Helsinki (2000). All patients were female and did not have any specific dermal pathologies or other systemic pathologies not pharmacologically controlled.

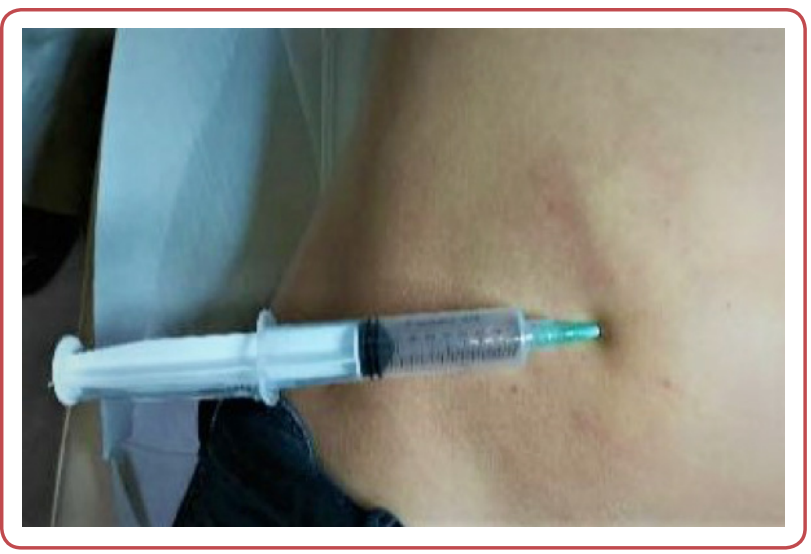

Figure 1: Extraction of adipose tissue with a syringe and a 16 G needle

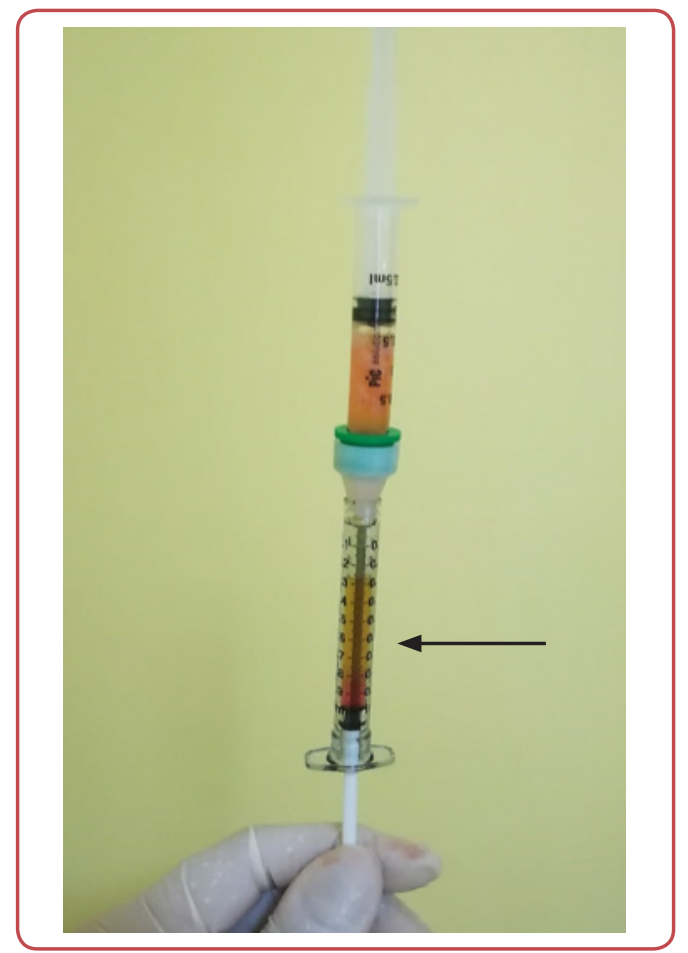

Figure 2: Microfiltration at 50 microns of adipose tissue after the Tonnard's 2013 disruption
For the extraction of the adipose tissue, a $10 \mathrm{~mL}$ syringe with luer lock connection combined with a 16 G needle (Figure 1) was used, while for filtration a 50-micron filter was used in order obtain vital progenitors in the suspension, by excluding fibrous shoots and cellular fragments. ${ }^{8}$ After performing a local anesthesia with Klein solution on the tissue-removal area, procedure involved the extraction of $3 \mathrm{~mL}$ of adipose tissue. The extraction with a needle allowed a survival of the progenitors, ${ }^{7}$ as well as an overlap in the quantity and quality of viable cells extracted versus the extraction with a multi-hole cannula, but with less trauma for the cells. ${ }^{21,22}$ After the extraction of the adipose tissue, the suspension was settled for 10 minutes to eliminate anesthesia fluids and, finally, $2 \mathrm{~mL}$ of fat was obtained.

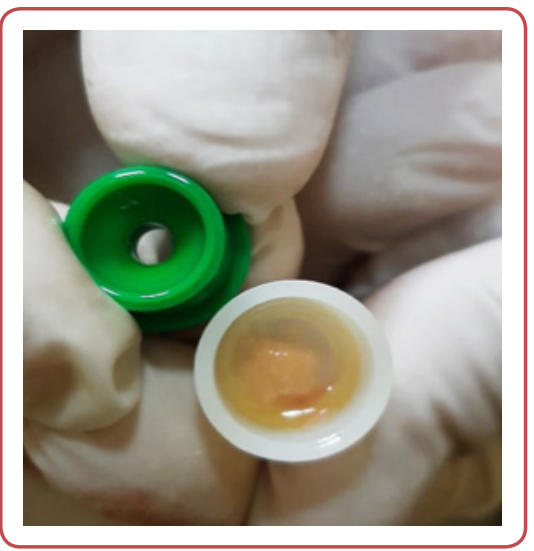

Figure 3: Fibrous shoots and cellular fragments by filter retained

At this point, the tissue was disrupted as indicated by Tonnard 2013, and it was filtered at $50 \mathrm{mi}-$ crons (Figure 2) to better preserve the SP, from which $0.8 \mathrm{~mL}$ of final suspension (Figure 2) was obtained. ${ }^{8}$ During the disintegration and filtration phase there is a loss of vital elements, but their therapeutic potential is higher because the fibrous shoots and cellular fragments are eliminated (Figure 3). 2, 21, 22 In fact, fibrous shoots and cellular fragments are responsible for the activation of inflammatory process in dermis through the Toll-like receptors expressed on dendridic cells and macrophages which increase in number during aging in dermis and it is incompatible with our regenerative procedure. ${ }^{7}$ We obtained the 5 mL STBA from a centrifuged peripheral venous blood sample (Figure 4) from which the buffy coat was excluded and gelled by denaturation through heat in a dry steriliser at $70{ }^{\circ} \mathrm{C}$ for 10 minutes (Figure 5), and it was left to cool. The scaffold was joined to the $0.8 \mathrm{~mL}$ vital suspension through a three-way tap with gentle movement 5-7 times (Figure 6). 


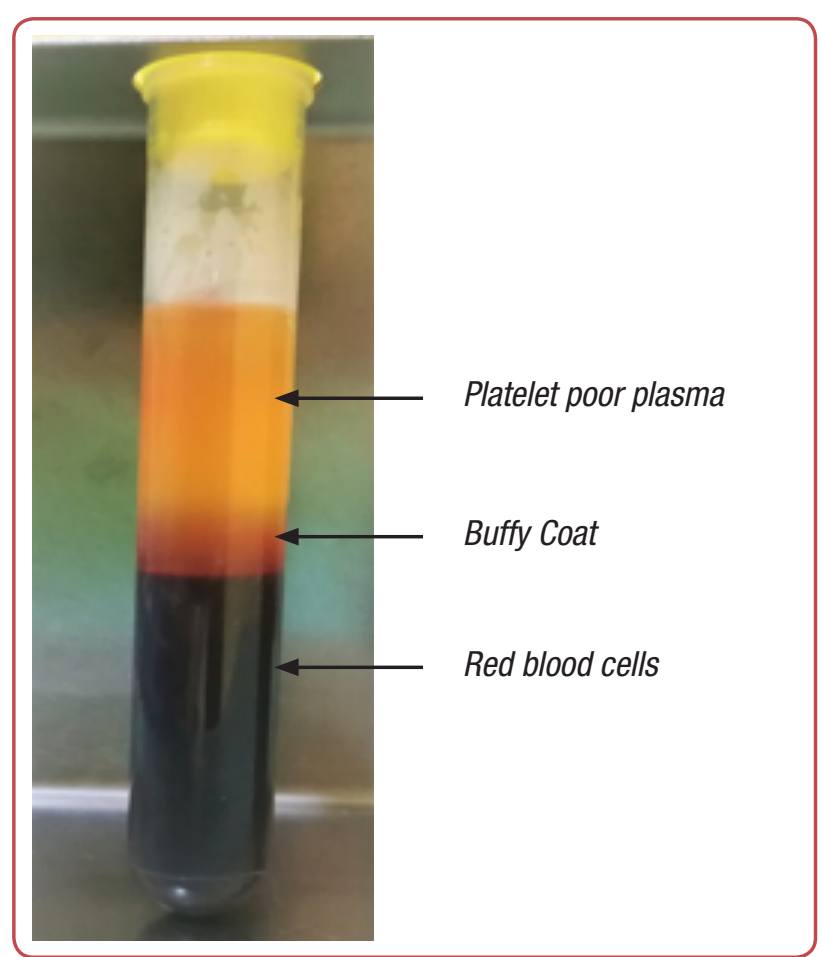

Figure 4: Peripheral blood centrifugation. The platelet poor plasma was gelled and the buffy coat was combined immediately before injecting the wrinkles and surrounding tissues

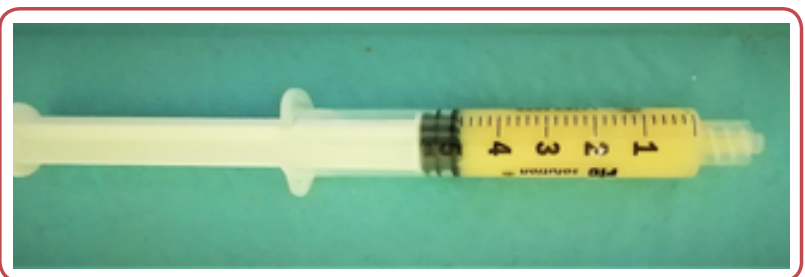

Figure 5: STBA, Autologous Biological Tissue Support. Plasma gel obtained in a dry oven at $70^{\circ} \mathrm{C}$ for 10 minutes and left to cool

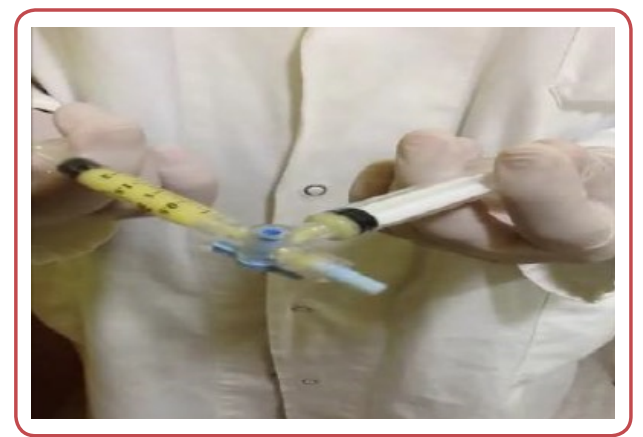

Figure 6: Mixing of autologous tissues through a three-way tap

The buffy coat was joined with the same threeway tap and with the same movements order to obtain a homogeneous gel immediately before injecting it into dermis (Figure 7). The suspension was injected into the deep dermis through a $25 \mathrm{G}$ needle and a microcannula. The whole procedure took 30 minutes for each patient.

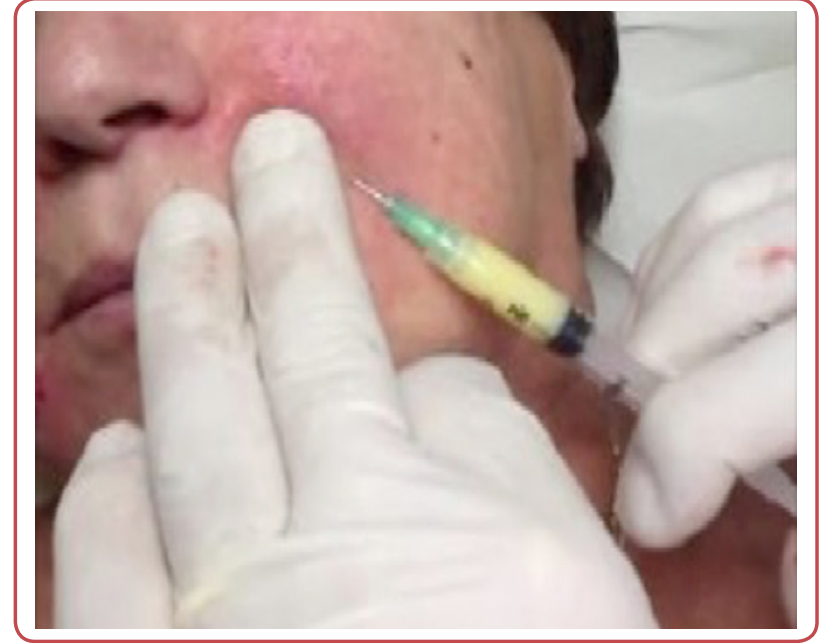

Figure 7: Correction of deep wrinkles and surrounding tissues

Subjects evaluated their satisfaction or dissatisfaction by giving scores on the appearance of firmness and wrinkles, by using a scale of 0 - 4 for each criteria ( 0 = unsatisfactory; 4 = satisfactory), as suggested by Berardesca et al, after the first and second month and a six-month follow-up. ${ }^{12}$

Table 1: Vancouver Modified Scale

\begin{tabular}{|c|c|c|c|}
\hline Baseline & & & $\begin{array}{l}\text { Follow-up } \\
60 \text { Days }\end{array}$ \\
\hline Vascularity: & Red & Purple & Normal \\
\hline Pigmentation: & Hyperpigmentation & Hypopigmentation & Normal \\
\hline Pliability: & Firm & Ropes & Normal/Supple \\
\hline
\end{tabular}

Individual signs of wrinkles and the degree of correction obtained for each treatment and each area were objectively evaluated by using a 10-0 visual analog scale (VAS) with separate scores for each site. Surrounding tissues were also evaluated with the Modified Vancouver scale (Table 1).

\section{Results}

Subjects evaluated their satisfaction or dissatisfaction by giving scores on the appearance of firmness and wrinkles, by using a Berardesca's scale. Results are reported in Figure 10.

With the VAS scale the results are shown in a Figure 11 ( $10=$ no correction; 5 = satisfactory correction; $0=$ complete correction). Figure 8 and figure 9 , represents photographic examples of results.

With this technique we were able to obtain excellent results. In fact, wrinkles were improved even 


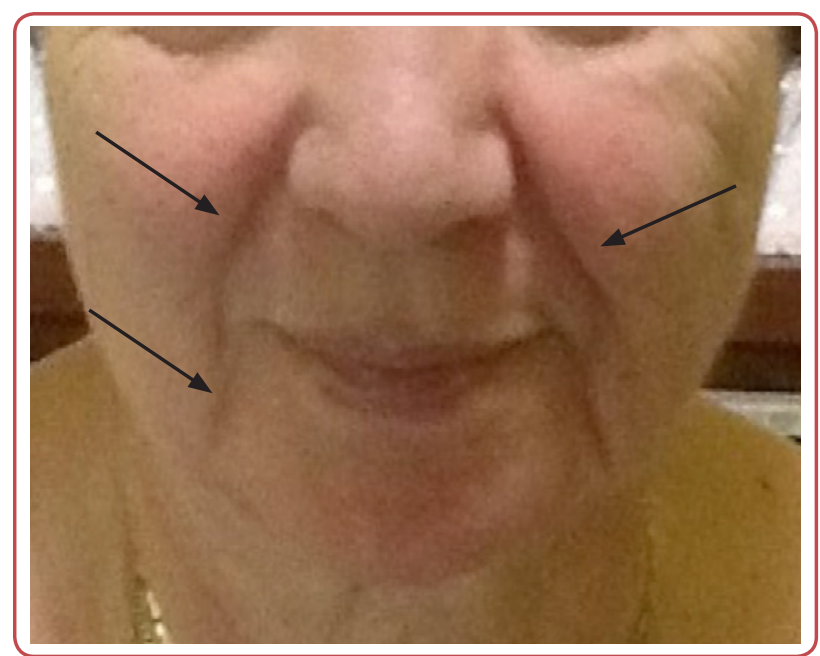

Figure 8: Before treatment

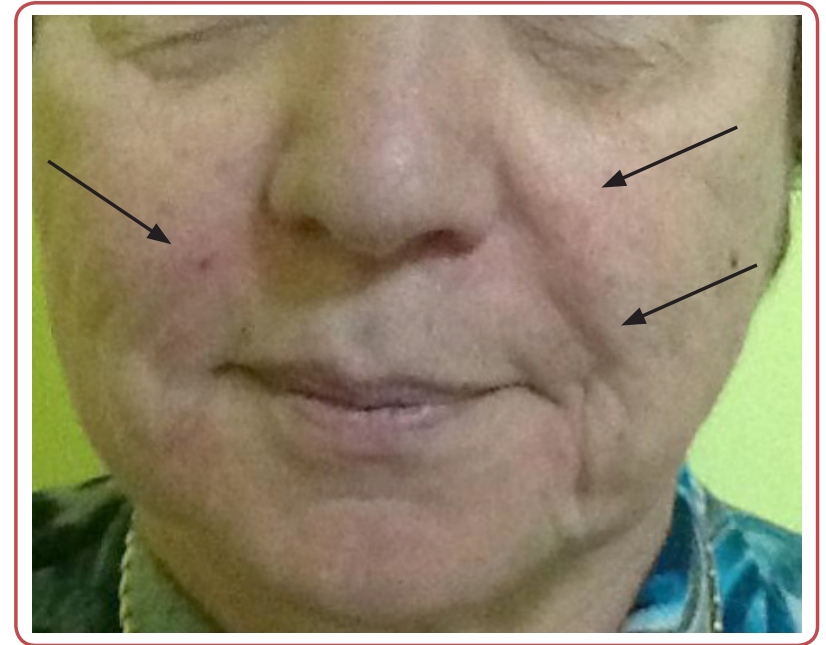

Figure 9: After treatment

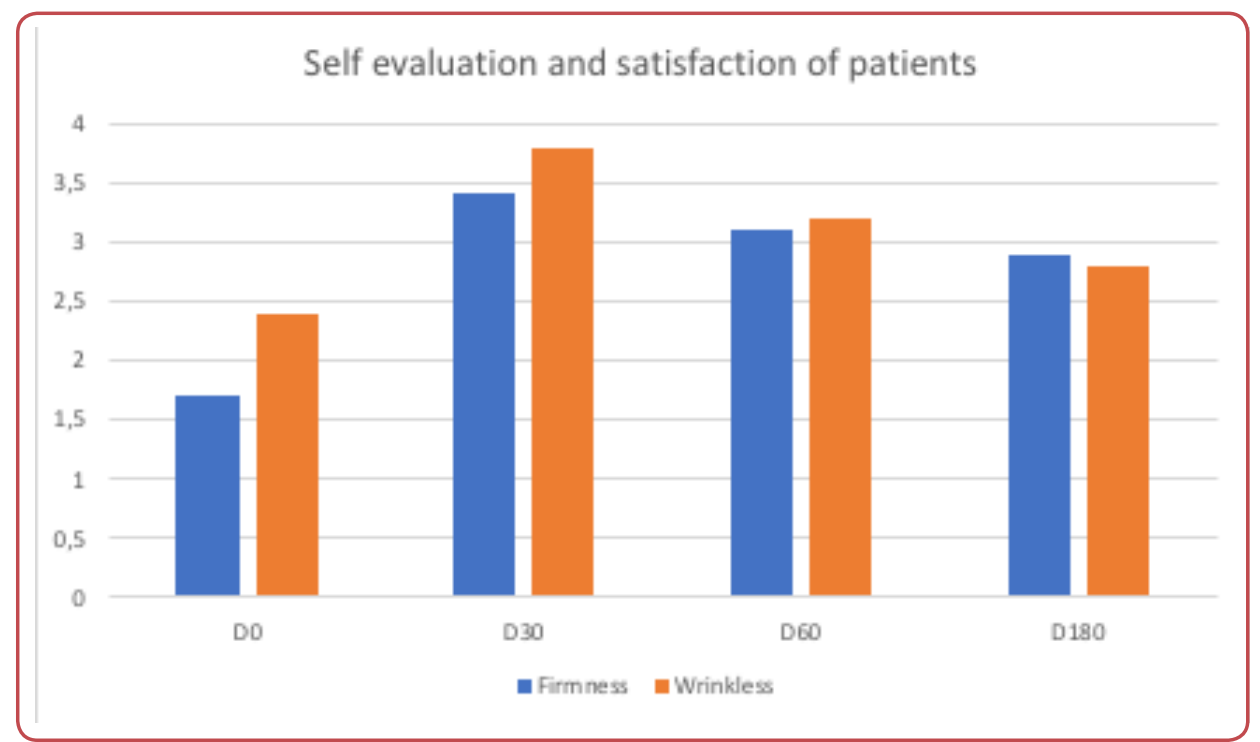

Figure 10: Self-evaluation and patient satisfaction based on Berardesca's Scale D0: after treatments, D30: after 30 days, D60: after 60 days, D180: after 180 days

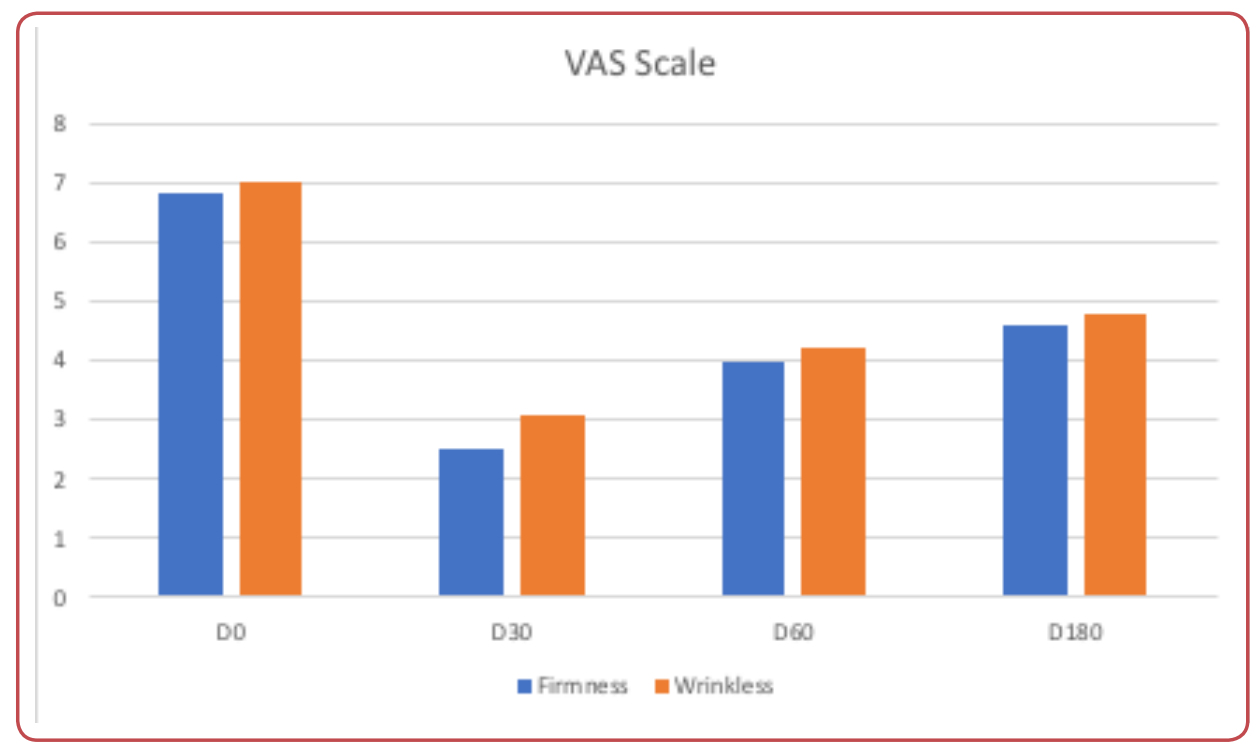

Figure 11: Evaluation based on visual analog scale (VAS)

DO: after treatments, D30: after 30 days, D60: after 60 days, D180: after 180 days 
after 60 days, as shown by VAS scale after the first and second month and Berardesca's scale after a six-month follow-up.

\section{Discussion}

Aim of this study was to clinically demonstrate that a viable tissue micrograft joined to a non-inflammatory STBA, to which the buffy coat was added immediately prior to dermal injection, could be used in regenerative medicine and for the correction of deep wrinkles. We had the idea that the union of the autologous materials, prepared with this technique, could allow the activation the Progenitors with MSCs characteristics through growth factors and buffy coat, by allowing a more regulated and lasting action due to the ability of the protective plasma gel, and an induction to a more regular plasticity through the activation of CDs 44, 73, 90 and 105.

The possibility of exploiting the ability to bind growth factors to CD 44 receptors order to normalize the extra cytoplasmic matrix through a physiological neocollagenogenesis and the $C D$ 73, CD 90 and CD 105 for the improvement of the vascularisation of the papillary dermis induced to enroll a sufficient number of patients and to study them for a suitable time, to be able to express a retrospective clinical evaluation in vivo. ${ }^{8,23}$

This procedure lasted for 30 minutes for each patient. All patients were satisfied with the treatment. The physical examination that the patients underwent during the follow-up was in line with their self-assessment.

There are no similar therapeutic approaches in the literature that combine the heat-gelled plasma used as a medium with a protective agent for the viable micrografts identified in the progenitors with the characteristics of MSCs and activated with growth factors joined immediately before infiltration to activate their tissue plasticity. With this technique we were able to obtain excellent results.

\section{Conclusion}

This prospective clinical evaluation allowed us to ascertain excellent results, that have been obtained with our method for the treatment of deep wrinkles and dermal surrounding tissues through a suspension of Progenitors with ADSCa in a not inflammatory STBA combined with buffy coat. This phenomenon can be biologically explained with a better regulation of the Progenitor Plasticity phenomenon through the signaling pathways of CD 44 of CD 73, CD 90 and CD 105. Furthermore, the STBA could physiologically mimic the formation of natural niches in which the adult mesenchymal stem cells are included, by providing them with a protective action and a longer stay at the injection site with beneficial effects on the regulation of ECM, neocollagenogenesis and neovasculogenesis. The illustrated method opens new therapeutic solutions in the regenerative correction of deep wrinkles and surrounding tissues with autologous materials. Moreover, this method can be performed by the physician very quickly and by providing extreme satisfaction to patients, by avoiding the inflammatory component of a nanofat consisting of fibrous shoots and cells fragments.

\section{Acknowledgements}

None.

\section{Conflict of interest}

None.

\section{References}

1. Lanza R, Rosenthal N. The stem cell challenge. Sci Am 2004 Jun;290(6):92-9.

2. Pontieri GM, Russo MA, Frati L, editors. General pathology. 4th edition. Padua: Piccin Editore, 2011. Italian. Pag 282;399-445;631.

3. Rong YH, Zhang GA, Wang C, Ning FG. Quantification of type I and III collagen content in normal human skin in different age groups. Zhonghua Shao Shang Za Zhi 2008 Feb;24(1):51-3. Chinese. 
4. Jelaska A, Strehlow D, Korn JH. Fibroblast heterogeneity in physiological conditions and fibrotic disease. Springer Semin Immunopathol 1999;21(4):385-95.

5. Svolacchia F, De Francesco F, Trovato L, Graziano A, Ferraro GA. An innovative regenerative treatment of scars with dermal micrografts. J Cosmet Dermatol 2016 Sep;15(3):245-53.

6. Tonnard P, Verpaele A, Peeters G, Hamdi M, Cornelissen $M$, Declercq $H$. Nanofat grafting: basic research and clinical applications. Plast Reconstr Surg 2013 Oct;132(4):1017-26.

7. Svolacchia F, Svolacchia L. Microfiltered vs only disaggregated mesenchymal stem cells from adipose tissue in regenerative medicine. Scr Med 2020;51(3):152-7.

8. Svolacchia F, Svolacchia L. Dermal regeneration with MilliGraft $®$ kit of nanofat: the micrograft of adipose tissue. A clinical assessment study. Scr Med 2019;50(3):117-21.

9. Maisel-Campbell AL, Ismail A, Reynolds KA, Poon E, Serrano L, Grushchak S, et al. A systematic review of the safety and effectiveness of platelet-rich plasma (PRP) for skin aging. Arch Dermatol Res 2020 Jul;312(5):301-315.

10. Gammon RR, Devine D, Katz LM, Quinley E, Wu Y, Rowe $\mathrm{K}$, et al. Buffy coat platelets coming to America: Are we ready? Transfusion $2021 \mathrm{Feb}$;61(2):627-633.

11. Xu P, Wu Y, Zhou L, Yang Z, Zhang X, Hu X, et al. Platelet-rich plasma accelerates skin wound healing by promoting re-epithelialization. Burns Trauma 2020 Aug 14;8:tkaa028. doi: 10.1093/burnst/tkaa028.

12. Berardesca E, Distante F, Anthoine P, Rabbiosi G, Aubert L. Clinical and instrumental evaluation of the activity of an anti-wrinkle product on cutaneous relief and photoaged skin. J Appl Cosmetol 1997;15:69-75.

13. Hass R, Kasper C, Böhm S, Jacobs R. Different populations and sources of human mesenchymal stem cells (MSC): A comparison of adult and neonatal tissue-derived MSC. Cell Commun Signal 2011 May 14;9:12. doi: 10.1186/1478-811X-9-12.

14. Chan AK, Heathman TR, Coopman K, Hewitt CJ. Multiparameter flow cytometry for the characterisation of ex- tracellular markers on human mesenchymal stem cells. Biotechnol Lett 2014;36(4):731-41.

15. Svolacchia F, Svolacchia L. Correction of defects in the flaws of Bone Tissue Facial, through the use of Tricalcium Phosphate or Calcium Hydroxyapatite with Plasma Gel. A clinical assessment study. J Appl Cosmetol 2017 (35):57-65.

16. Karamichos D, Hutcheon AE, Zieske JD. Transforming growth factor- $\beta 3$ regulates assembly of a non-fibrotic matrix in a 3D corneal model. J Tissue Eng Regen Med 2011 Aug;5(8):e228-38.

17. Zhao X, Liu Y, Jia P, Cheng H, Wang C, Chen S, et al. Chitosan hydrogel-loaded MSC-derived extracellular vesicles promote skin rejuvenation by ameliorating the senescence of dermal fibroblasts. Stem Cell Research \& Therapy (preprint). Available from: https://www. researchsquare.com/article/rs-118943/v1 (Cited: 24Feb-21).

18. Croce MA, Dyne K, Boraldi F, Quaglino D Jr, Cetta G, Tiozzo R, Pasquali Ronchetti I. Hyaluronan affects protein and collagen synthesis by in vitro human skin fibroblasts. Tissue Cell 2001 Aug;33(4):326-31.

19. Song HF, Chai JK, Lin ZH, Liu NF, Chen ML, Zhao YZ, et al. Expression of hyaluronic acid and its receptor in the process of wound healing in different skin tissues and its significance. Zhonghua Yi Xue Za Zhi 2003; 83:1070-4.

20. Qiao J, An N, Ouyang X. Quantification of growth factors in different platelet concentrates. Platelets 2017 Dec;28(8):774-778.

21. Bi HS, Zhang C, Nie FF, Pan BL, Xiao E. Basic and clinical evidence of an alternative method to produce vivo nanofat. Chin Med J (Engl) 2018 Mar 5;131(5):588-593.

22. Lo Furno D, Tamburino S, Mannino G, Gili E, Lombardo G, Tarico MS, et al. Nanofat 2.0: experimental evidence for a fat grafting rich in mesenchymal stem cells. Physiol Res 2017 Sep 22;66(4):663-671.

23. Aya KL, Stern R. Hyaluronan in wound healing: rediscovering a major player. Wound Repair Regen 2014 SepOct;22(5):579-93. 